\title{
Hyperparathyroidism-Jaw Tumor Syndrome
}

\author{
Travis D. Weaver Mohamed K.M. Shakir Thanh D. Hoang \\ Division of Endocrinology, Department of Medicine, Walter Reed National Military Medical \\ Center, Bethesda, MD, USA
}

\section{Keywords}

Jaw tumor $\cdot$ Hyperparathyroidism

\begin{abstract}
Primary hyperparathyroidism is a relatively common endocrine disorder, affecting 7 out of 1,000 adults. The median age at onset is the 6 th decade of life. Our objective was to present a young patient with primary hyperparathyroidism who has a positive $C D C 73$ mutation. $A$ 23-year-old woman was evaluated for hypercalcemia that was found after surgery for bilateral ovarian cyst removal. Her family history included multiple family members with nephrolithiasis. The physical examination revealed a well-appearing Caucasian woman with no palpable neck mass. The laboratory results showed serum calcium at $11.7 \mathrm{mg} / \mathrm{dL}$ (ref. 8.4-10.2), ionized calcium at $1.44 \mathrm{mmol} / \mathrm{L}$ (ref. 1.12-1.32), and serum PTH at $192 \mathrm{pg} / \mathrm{mL}$ (ref. 11-65). A technetium-99 sestamibi scan revealed focal uptake inferior to the left thyroid lobe. Thyroid ultrasound showed a left parathyroid adenoma. The patient subsequently underwent left inferior parathyroidectomy, which confirmed parathyroid adenoma, with resultant normalization of serum calcium and PTH levels. Due to her young age at diagnosis, genetic testing was performed, which revealed a mutation of $C D C 73$. Although penetrance and expression are variable, the CDC73 mutation is associated primarily with hyperparathyroidism-jaw tumor syndrome, familial isolated hyperparathyroidism, and sporadic parathyroid carcinoma. This patient currently has no evidence of jaw, uterine or renal tumors on screening imaging. Given the potential impact of inheritable neoplasia, all young patients with unexplained hyperparathyroidism should be considered for genetic screening.

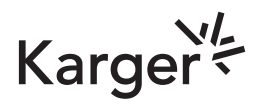




\section{Introduction}

Primary hyperparathyroidism (PHPT) is a common endocrine disorder. The prevalence is estimated to be $8-23$ cases per 10,000 in the USA, with a considerable predominance for women at a female-to-male ratio of 4:1. Many patients present in the 6th decade of life [1]. When PHPT is found in younger patients there is often an underlying genetic mutation.

\section{Methods}

The diagnostic studies include serum intact PTH, serum calcium, ionized calcium, neck ultrasound, technetium-99 sestamibi scanning, and genetic testing.

\section{Case Report}

A 23-year-old woman was referred to endocrinology for evaluation of hypercalcemia that was found after surgery for bilateral ovarian cyst removal. At the time of presentation, she felt well and had no complaints. Her medical history is otherwise unremarkable. Her family history includes multiple family members with nephrolithiasis. Physical examination revealed a well-appearing Caucasian woman with no palpable neck mass. The initial laboratory evaluation showed a serum calcium level of $11.7 \mathrm{mg} / \mathrm{dL}$ (8.4-10.2), ionized serum calcium at $1.44 \mathrm{mmol} / \mathrm{L}$ (1.12-1.32), and serum PTH at $192 \mathrm{pg} / \mathrm{mL}$ (11-65). Technetium-99 sestamibi scanning was performed, which revealed a low attenuating mass with focal and persistent uptake just inferior to the left thyroid lobe (Fig. 1). Thyroid ultrasound also confirmed a left parathyroid adenoma (Fig. 2). The patient subsequently underwent left inferior parathyroidectomy, which showed parathyroid adenoma (Fig. 3), with resultant normalization of her serum calcium and PTH levels. Due to her young age at diagnosis, genetic testing was performed, which revealed a mutation of $C D C 73$. Jaw X-ray resulted normal.

\section{Discussion}

When first described in the 1930s, PHPT was a severe disease with multiple significant implications for the end organs. Today, in well-resourced health systems, where biochemical testing is common, PHPT is more frequently found incidentally on laboratory evaluation. A

1

Fig. 1. Sestamibi CT scan showing a low attenuating mass with focal and persistent uptake just inferior to the left thyroid lobe.

Fig. 2. Thyroid ultrasound also confirmed a left parathyroid adenoma.
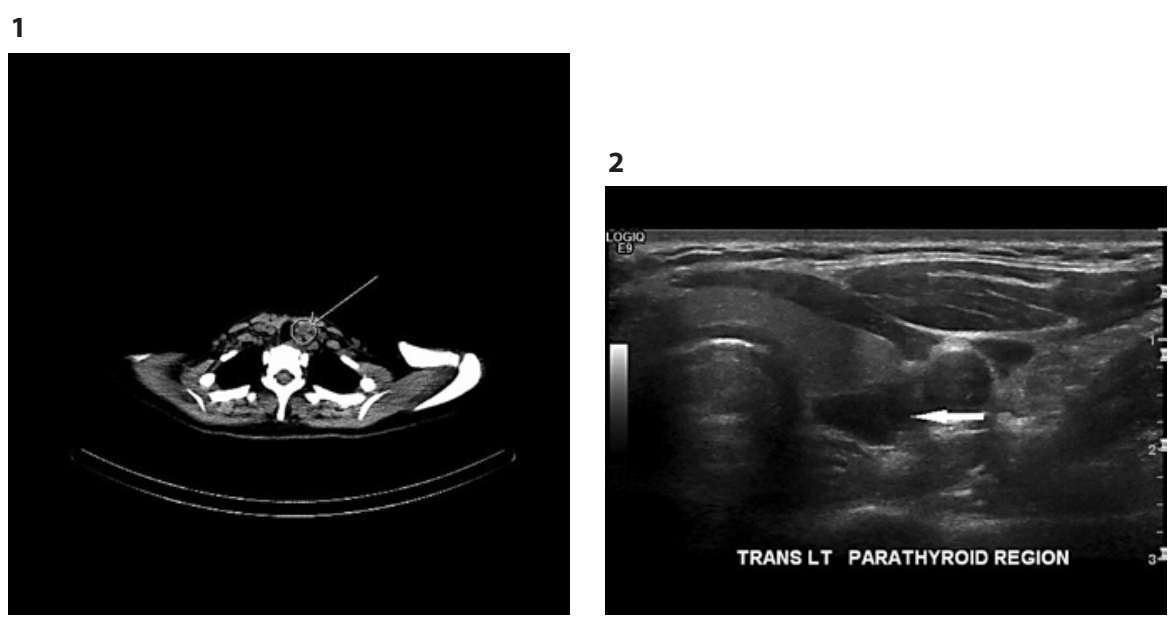
Fig. 3. Histology confirmed a parathyroid adenoma.

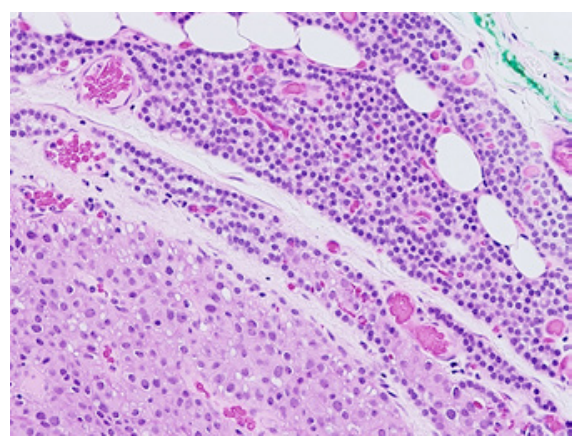

single benign adenoma is found in approximately $80 \%$ of cases of PHPT. Underlying inheritable genetic mutation may be seen in up to $10 \%$ of patients. Familial hyperparathyroidism is a clinically and genetically heterogeneous group of syndromes and includes multiple endocrine neoplasm type 1 (MEN1) and type 2A (MEN2A), familial isolated hyperparathyroidism, familial hypocalciuric hypercalcemia, and hyperparathyroidism-jaw tumor syndrome (HPTJT). Among these, it is often difficult to distinguish between isolated familial hyperparathyroidism and HPT-JT. However, mutations of the $C D C$ gene and other clinical features may be helpful in distinguishing between these two disorders [2,3].

HPT-JT is caused by germline mutations of the cell division cycle 73 (CDC73) gene that encodes the parafibroma, an amino acid protein with antiproliferative action. Although $75 \%$ of patients with HPT-JT have germline mutations within the coding region, 25\% of HPT-JT patients may have abnormalities in $C D C 73$ promoter regions, whole-exon or gene deletions, or mutations in epigenetic modifications or in unidentified genes [4-7]. This syndrome classically presents with parathyroid adenoma, ossifying tumors of the mandible, and renal or uterine neoplasms [5]. The mutation is inherited in an autosomal dominant pattern, and family history plays a key role in the diagnosis of this rare condition. Early recognition and treatment are important as the hypercalcemia tends to be more severe than with spontaneous parathyroid adenomas or MEN1 [6]. A single parathyroid adenoma is the most common presentation, and surgical resection is often curative of hyperparathyroidism [4, 7]. Our patient had an allele mutation associated with HPT-JT, a young age at onset for hyperparathyroidism, and a family history suggestive of an inherited calcium metabolism disorder. Her hypercalcemia and hyperparathyroidism quickly resolved after the parathyroidectomy.

Patients with HPT-JT may have associated uterine tumors, jaw tumors, and renal abnormalities. Tumors of the uterus, both benign and malignant, are the second most common manifestation, present in up to $50 \%$ of women with $C D C 73$ mutation. The most frequent presentation is menorrhagia, but women may also present with a history of miscarriage. Many patients are treated with hysterectomy at an early age. Women should undergo routine gynecologic care and pelvic imaging when clinically indicated at the onset of menarche, although there is no established guideline on this [4]. In this case, the patient had symptoms of menorrhagia and no evidence of uterine tumor on pelvic ultrasonography. Although there is no well-established guideline, it is suggested that family members with CDC73 mutations undergo periodic screening for hyperparathyroidism, jaw tumors, renal lesions, and for CDC73-related uterine disorders [4]. The so-called jaw tumors in HPT-JT occur is $30-50 \%$ of cases of HPT-JT. Most tumors are ossifying fibromas of the mandible or maxilla and occur more frequently in older patients but may present as early as the 3rd decade of life. Tumors may be resected based on size, location, and symptoms related to the lesion. Continued surveillance after resection is needed, since lesions are known to recur [4-7]. Our patient underwent an evaluation for jaw tumor, which was negative.

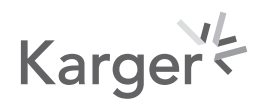


Up to $15 \%$ of cases with HPT-JT have renal involvement. Lesions may be unilateral or bilateral. Renal cysts are the more common finding; however, renal hamartomas and tumors have been described. For this reason, some experts have recommended that patients undergo surveillance with renal ultrasound, CT, or magnetic resonance imaging the time of diagnosis and at least every 5 years if initial imaging is normal [4]. We also evaluated our patient with a renal ultrasound, which was negative.

In patients with early-onset hyperparathyroidism and a family history suggestive of a disorder of calcium metabolism, genetic screening is an important diagnostic step for two reasons. First, although the overall incidence of inherited genetic disorder is low, the incidence is much higher among patients that do not fit the typical epidemiological pattern of PHPT, specifically young male patients with a family history of PHPT. Second, genetic mutation has a significant implication for a patient's risk of malignancy. For example, parathyroid carcinoma represents less than $1 \%$ of cases of isolated PHPT, but may be present in up to $18 \%$ of patients with $C D C 73$ mutation.

This patient currently has no evidence of jaw, uterine or renal tumors on screening imaging; however, the penetrance of this mutation is known to be variable, and up to $10 \%$ of patients have no clinical manifestation. Additionally, manifestations of disease appear to be less common in females. Ovarian tumors have been described with $C D C 73$ mutation with and without HPT-JT. This patient may actually have an underrecognized phenotype of HPT-JT with ovarian tumors which has only been described in case reports [8].

In conclusion, screening for $C D C 73$ germline mutations should be performed in patients with a family history of PHPT with relatively early-age onset ( $<40$ years), cystic parathyroid lesions, multiglandular involvement, parathyroid carcinoma, or the presence of ossifying jaw fibroma, uterine tumors or renal lesions [4, 9]. In patients with CDC73 mutation, surveillance screening should be performed to assess for other associated neoplasms.

\section{Statement of Ethics}

This paper has been cleared by the institutional review board. The patient has given written informed consent to publish the case (including publication of images).

\section{Conflict of Interest Statement}

The authors have no multiplicity of interest to disclose. The views expressed in this article are those of the authors and do not reflect the official policy of the Department of Army/Navy/Air Force, Department of Defense, or US Government.

\section{Funding Sources}

No funding was received.

\section{Author Contributions}

All individuals who qualify as authors have been listed; each has participated in the conception and design of this work, the analysis of data, the writing of the document, and the approval of the submission of this version. T.D. Weaver wrote the draft. M.K.M. Shakir and T.D. Hoang reviewed and critically edited the paper.

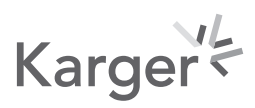




\section{References}

1 Yeh MW, Ituarte PH, Zhou HC, Nishimoto S, Liu IL, Harari A, et al. Incidence and prevalence of primary hyperparathyroidism in a racially mixed population. J Clin Endocrinol Metab. 2013;98(3):1122-9.

2 Bilezikian JP. Primary hyperparathyroidism. J Clin Endocrinol Metab. 2018;103(11):3993-4004.

3 Simonds WF. Genetics of hyperparathyroidism, including parathyroid cancer. Endocrinol Metab Clin North Am. 2017;46(2):405-18.

4 Torresan F, Iacobone M. Clinical features, treatment and surveillance of hyperparathyroidism-jaw tumor syndrome: an up-to-date and review of the literature. Int J Endocrinol. 2019;2019:1761030.

5 van der Tuin K, Tops CMJ, Adank MA, Cobben JM, Hamdy NAT, Jongmans MC, et al. CDC73-related disorders: clinical manifestations and case detection in primary hyperparathyroidism. J Clin Endocrinol Metab. 2017; 102(12):4534-40.

6 Chen JD, Morrison C, Zhang C, Kahnoski K, Carpten JD, Teh BT. Hyperparathyroidism-jaw tumour syndrome. J Intern Med. 2003;253(6):634-42.

7 du Preez H, Adams A, Richards P, Whitley S. Hyperparathyroidism jaw tumor syndrome: a pictoral review. Insights Imaging. 2016;7(6):793-800.

8 Sirbiladze RL, Uyar D, Geurts JL, Shaker JL. Ovarian granulosa cell tumor in a patient with a pathogenic variant in the CDC73 gene (hyperparathyroidism-jaw tumor syndrome). AACE Clin Case Rep. 2019;5(3):e222-5.

9 Iacobone M, Carnaille B, Palazzo FF, Vriens M. Hereditary hyperparathyroidism - a consensus report of the European Society of Endocrine Surgeons (ESES). Langenbecks Arch Surg. 2015;400(8):867-86. 